\title{
Fadiga clinicamente relevante em mulheres com câncer de mama: prevalência e fatores associados
}

\author{
Daniela de Araujo Lamino² \\ Cibele Andruciolli de Mattos Pimenta \\ Patrícia Emilia Braga ${ }^{4}$ \\ Dálete Delalibera Corrêa de Faria Mota5
}

doi:10.11144/Javeriana.IE17-1.fcrm

Como citar: Lamino DA, Pimenta CAM, Braga PE, Mota DDCF. Fadiga clinicamente relevante em mulheres com câncer de mama: prevalência e fatores associados. Investig Enferm. Imagen Desarr. 2015;17(1):65-76. http://dx.doi.org/10.11144/Javeriana.IE17-1.fcrm

1. Articulo original de investigación. Recebido: 5 de junho de 2014. Aceito: 1 de setembro de 2014. Artigo extraído da dissertação "Prevalência e fatores associados à fadiga em mulheres com câncer de mama”, apresentada à Escola de Enfermagem da Universidade de São Paulo, Brasil, em 2012.

2. Mestre em Ciências pela Escola de Enfermagem da Universidade de São Paulo, Brasil. Bolsista CAPES. Enfermeira de Pesquisa Clinica no Instituto do Câncer do Estado de São Paulo. Correio eletrônico: danilamino@yahoo.com.br

3. Professora titular do Departamento de Enfermagem Médico Cirúrgica, Universidade de São Paulo, Brasil. Correio eletrônico: parpca@usp.br

4. Epidemiologista, Escola de Enfermagem da Universidade de São Paulo, Brasil. Doutorada em Saúde Pública, São Paulo, Brasil. Correio eletrônico: bragapati@netpoint.com.br.

5. Professora-doutora, Faculdade de Enfermagem, Universidade Federal de Goiás, Brasil. Correio eletrônico: dalete.mota@globo.com 


\section{Resumo}

Introdução: A fadiga é um sintoma prevalente em pacientes com câncer. Objetivo: O estudo analisou a prevalência e os preditores independentes de fadiga em mulheres com câncer de mama. Material e método: Trata-se de estudo transversal com amostra não probabilística de 163 pacientes em acompanhamento ambulatorial. Fadiga foi avaliada pela Escala de Fadiga de Piper. Resultados: Fadiga clinicamente relevante (escore $\geq 4$ ) esteve presente em $31,9 \%$ da amostra e a intensidade média foi 6,0 (DP $=1,3$ ). Dor e depressão foram fatores independentemente associados à fadiga. Conclusões: A associação fadiga, dor e depressão confirmou a existência de cluster de sintomas. O controle da fadiga é pouco conhecido, mas depressão e dor podem ser tratadas e talvez, proporcionar alivio da fadiga.

Palavras-chave: fadiga; prevalência; fator de risco; neoplasia da mama; cuidados paliativos

\section{Fatiga clínicamente relevante en las mujeres con cáncer de mama: prevalencia y factores asociados}

\section{Resumen}

Introducción: La fatiga es un síntoma frecuente en pacientes con cáncer. Objetivo: Examinar la prevalencia y los predictores independientes de la fatiga en las mujeres con cáncer de mama. Material e método: Se trata de un estudio transversal con una muestra no probabilística de 163 pacientes en acompañamiento ambulatorio. La fatiga fue evaluada por la Escala de Fatiga de Piper. Resultados: La fatiga clínicamente relevante (puntuación $\geq 4$ ) estuvo presente en el 31,9\% de la muestra y la intensidad media fue de $6(\mathrm{DP}=1,3)$. El dolor y la depresión fueron factores independientemente asociados con la fatiga. Conclusiones: La asociación de la fatiga, el dolor y la depresión confirmaron la existencia de clúster de síntomas. El control de la fatiga es poco conocido, pero la depresión y el dolor pueden ser tratados y tal vez proporcionar alivio de la fatiga.

Palabras clave: fatiga; prevalencia; factores de riesgo; cáncer de mama; cuidados paliativos 


\section{Clinical Relevant Fatigue in Women with Breast Cancer: Prevalence and Associated Factors}

\section{Abstract}

Introduction: Fatigue is a common symptom in patients with cancer. Objective: To examine the prevalence and independent predictors of fatigue in women with breast cancer. Material and Method: A cross-sectional study with a nonrandom sample of 163 patients in outpatient follow-up. Fatigue was assessed by the Piper Fatigue Scale. Results: Clinical relevant fatigue (score $\geq 4$ ) was present in $31.9 \%$ of the sample and the average intensity was $6(\mathrm{SD}=1.3)$. Pain and depression were factors independently associated with fatigue. Conclusions: The association of fatigue, pain and depression confirmed the existence of cluster of symptoms. The management of fatigue is poorly understood, but depression and pain may be treated and may provide relief from fatigue.

Keywords: fatigue; prevalence; risk factors; breast cancer; hospice 


\section{Introdução}

O câncer de mama é a neoplasia maligna mais freqüente entre mulheres no mundo e dentre dos sintomas que o acompanha, destaca-se a fadiga, sintoma ainda pouco explorado na população brasileira. Vários fatores contribuem para a fadiga relacionada ao câncer, incluindo aqueles relacionados ao tumor, aos aspectos psicossociais, a outras morbidades e sintomas $(1,2)$.

A prevalência de fadiga em doentes com câncer de mama pode variar de acordo com a fase da doença ou tratamento realizado, podendo variar entre $30 \%$ e $70 \%$ (3), e, mesmo após a conclusão do tratamento, pode acometer aproximadamente um terço das pacientes (2).

No câncer de mama tem sido investigada a relação de fadiga com sintomas físicos, psicológicos, características do ambiente, características sócio-demográficas, tratamento, medicamentos, alterações fisiológicas e genéticas (4-6). No entanto, os estudos apresentam resultados contraditórios.

Na literatura internacional, a prevalência e os fatores associados à fadiga em pacientes com câncer têm sido explorados, no entanto, na literatura brasileira, existem poucas publicações e pouco se sabe sobre a prevalência e quais os elementos que contribuem para a ocorrência do sintoma em nossa população, o que justifica a realização da presente pesquisa.

\section{Objetivo}

Analisar a prevalência e os preditores independentes relacionados à fadiga em mulheres com câncer de mama.

\section{Método}

Trata-se de estudo transversal. Os dados são oriundos de banco de dados cujos dados foram coletados na cidade de São Paulo, no período de julho de 2006 a abril de 2008, em três serviços de oncologia (Clínica de Oncologia Médica Privada, Ambulatório de Oncologia do Hospital Brigadeiro e Clínica de Oncologia Médica do Hospital Santa Helena).

A amostra, não probabilística, foi constituída por 163 mulheres com câncer de mama em acompanhamento ambulatorial. Os critérios de inclusão foram idade superior a 18 anos, escolaridade acima de quatro anos de estudo, capacidade de comunicação e compreensão preservadas e ausência de infecção aguda no momento da coleta dos dados.

A fadiga foi avaliada através da Escala de Fadiga de Piper-Revisada (0-10), escala validada em 2009 (7), apresentando alpha de Cronbach igual a 0,94 . Dor e de prejuízo do sono foram avaliados por Escala Visual Numérica (0-10), a capacidade funcional por meio da Escala de Karnofsky (8), podendo variar de 10 a 100, e depressão foi avaliada por meio do Inventário de Depressão de Beck (0-63), validado para a língua portuguesa por Gorenstein e Andrade (9). 
Fadiga foi classificada em três níveis (leve, moderada e intensa), de acordo com a distribuição dos escores em quartis (quartil 1: 3,2; quartil 2: 4,8; quartil 3: 6,2) e o consenso sobre fadiga do National Comprehensive Cancer Network (10) que, considera o escore de fadiga $\geq 4$ como evento clinicamente significativo quando avaliada em Escala Numérica de 0 a 10. Os escores que variaram entre 0,1 e 3,9 foram classificados em fadiga leve; de 4 a 5,9, moderada, e de 6 a 10, intensa. Em seguida, calculou-se a prevalência de fadiga e seu respectivo intervalo com $95 \%$ de confiança.

Em estudos de corte transversal a relação entre exposição e desfecho é estimada pela razão de prevalência (RP). Quando a prevalência é alta, o OR não é uma boa aproximação da RP e seu uso é inadequado $(11,12)$. Considerando a alta prevalência de fadiga (escore $\geq 4$ ) no presente estudo (31,9\%), estimou-se a RP e seus respectivos intervalos de confiança (IC 95\%) à análise univariada.

Considerando as variáveis dependentes na forma quantitativa, empregou-se o teste $\mathrm{T}$ de Student ou o teste não paramétrico de MannWhitney, após verificação de não normalidade pelo teste não paramétrico de Kolmogorov-Smirnov.

Para identificar as variáveis independentemente associadas à fadiga deu-se continuidade à análise com a regressão múltipla de Cox com variância robusta (13). Em estudos de corte transversal com alta prevalência, este modelo mostrou-se como alternativa mais adequada à regressão logística (14). Nessa etapa utilizaram-se todas as variáveis que apresentaram valores de $p$ menores que 0,25 à análise univariada com as variáveis na forma qualitativa. As análises, descritiva e inferencial, foram realizadas no programa estatístico Stata, versão 9.0 e adotou-se o nivel de significância de 5\%.

\section{Aspectos éticos}

O estudo foi submetido e autorizado pelo Comitê de Ética da Escola de Enfermagem da Universidade de São Paulo (Processo 511/2005/CEP-EEUSP), Comitês do Hospital Santa Helena e do Hospital Brigadeiro. As pacientes foram convidadas a participar dos estudos, e aquelas que aceitaram, assinaram o Termo de Consentimento Esclarecido em duas vias.

\section{Resultados}

Dentre as mulheres com algum grau de fadiga, ou seja, escore entre 0,1 a 10 (49,7\% da amostra total do estudo), o sintoma teve intensidade de moderada a intensa em $64,2 \%$ das mulheres com essa morbidade. Nesse estrato, a intensidade média de fadiga foi de 6,0 $(\mathrm{DP}=1,3$; mediana $=6,0$; mínimo = 4; máximo =9). Nenhuma das pacientes apresentou o escore máximo de fadiga (10). Considerando o escore de fadiga $\geq 4$ como ocorrência de tal evento, obteve-se, para a amostra em estudo, uma prevalência do sintoma de $31,9 \%$ (tabela 1 ). 
TABela 1. Razão de prevalência de fadiga segundo variáveis sócio-demográficas (São Paulo, 2007-2008)

\begin{tabular}{|c|c|c|c|c|}
\hline Variáveis & Total & $\begin{array}{c}\text { Fadiga } \\
\text { n. }{ }^{\circ} \text { (preva- } \\
\text { lência) }\end{array}$ & $\begin{array}{l}\text { RP (IC } \\
\mathbf{9 5 \% )}\end{array}$ & Valor de $\mathbf{p}$ \\
\hline Idade (anos) & & & & 0,620 \\
\hline$\leq 45$ & 48 & $18(37,5)$ & 1,00 & \\
\hline 46 a 64 & 98 & $29(29,6)$ & $\begin{array}{r}0,79(0,49- \\
1,27)\end{array}$ & \\
\hline$\geq 65$ & 16 & $5(31,3)$ & $\begin{array}{r}0,83(0,37- \\
1,88)\end{array}$ & \\
\hline $\begin{array}{c}\text { Escolaridade (anos de estudo; } \mathbf{n} \\
=161 \text { ) }\end{array}$ & & & & 0,572 \\
\hline 6 a 8 & 13 & $4(30,8)$ & 1,00 & \\
\hline 9 a 11 & 51 & $19(37,3)$ & $\begin{array}{r}1,21(0,50- \\
2,96)\end{array}$ & \\
\hline$\geq 12$ & 97 & $28(28,9)$ & $\begin{array}{r}0,94(0,39- \\
2,25)\end{array}$ & \\
\hline Renda familiar em SM (n = 147) & & & & 0,947 \\
\hline 1 a 5 & 41 & $14(34,2)$ & 1,00 & \\
\hline 6 a 10 & 35 & $12(34,3)$ & $\begin{array}{r}1,00(0,54- \\
1,88)\end{array}$ & \\
\hline 11 a 20 & 39 & $13(33,3)$ & $\begin{array}{r}0,98(0,53- \\
1,81)\end{array}$ & \\
\hline$>20$ & 32 & $9(28,1)$ & $\begin{array}{r}0,59(0,41- \\
1,66)\end{array}$ & \\
\hline Com companheiro $(n=160)$ & & & & 0,839 \\
\hline Não & 72 & $24(33,3)$ & 1,00 & \\
\hline Sim & 88 & $28(31,8)$ & $\begin{array}{r}0,95(0,61- \\
1,50)\end{array}$ & \\
\hline Trabalho remunerado $(n=162)$ & & & & 0,269 \\
\hline Não & 102 & $36(35,6)$ & 1,00 & \\
\hline Sim & 60 & $16(26,7)$ & $\begin{array}{r}0,76(0,46- \\
1,24)\end{array}$ & \\
\hline Serviço de atendimento $(n=163)$ & & & & 0,340 \\
\hline Público & 28 & $11(39,3)$ & 1,00 & \\
\hline Privado & 135 & $41(30,4)$ & $\begin{array}{r}0,77(0,46- \\
1,31) \\
\end{array}$ & \\
\hline
\end{tabular}

RP: razão de prevalência; SM: salário mínimo (considerando que um salário mínimo era igual a $\mathrm{R} \$ 380,00$ no ano de 2007). 
Nenhuma das variáveis sócio-demográficas analisadas apresentou associação com fadiga (tabela 2).

TABEla 2. Razão de prevalência de fadiga segundo variáveis relacionadas ao tumor e à terapêutica (São Paulo, 2007-2008)

\begin{tabular}{|c|c|c|c|c|}
\hline Variáveis & Total & $\begin{array}{c}\text { Fadiga } \\
\text { n. }{ }^{\circ} \text { (pre- } \\
\text { valência) } \\
\end{array}$ & RP (IC 95\%) & Valor de $p$ \\
\hline $\operatorname{IMC}(n=158)$ & & & & 0,360 \\
\hline 18,5 a 24,9 & 73 & $20(27,4)$ & 1,00 & \\
\hline 25,0 a 29,9 & 59 & $23(39,0)$ & $0,70(0,43-1,15)$ & \\
\hline$\geq 30,0$ & 26 & $8(30,8)$ & $0,79(0,41-1,53)$ & \\
\hline Hemoglobina (g/d1; $n=145$ ) & & & & 0,030 \\
\hline 12 a 16 & 109 & $31(28,4)$ & 1,00 & \\
\hline$<12$ & 36 & $17(47,2)$ & $1,66(1,05-2,62)$ & \\
\hline Estadiamento clinico $(n=124)$ & & & & 0,082 \\
\hline I & 31 & $5(16,1)$ & 1,00 & \\
\hline II ou III ou IV & 93 & $32(34,4)$ & $2,13(0,91-5,01)$ & \\
\hline Tratamento atual $(n=161)$ & & & & 0,745 \\
\hline Não estava em tratamento & 56 & $18(32,1)$ & 1,00 & \\
\hline Quimioterapia & 56 & $18(32,1)$ & $1,00(0,58-1,72)$ & \\
\hline Hormonioterapia & 38 & $11(28,9)$ & $0,90(0,48-1,69)$ & \\
\hline Outros tratamentos ${ }^{*}$ & 11 & $5(45,4)$ & $1,41(0,67-3,00)$ & \\
\hline
\end{tabular}

RP: razão de prevalência

${ }^{*} \mathrm{QT}+\mathrm{RT}(\mathrm{n}=3) ; \mathrm{QT}+\mathrm{HT}(\mathrm{n}=1) ; \mathrm{RT}+\mathrm{HT}(\mathrm{n}=1) ;$

$\mathrm{HT}+\mathrm{IT}(\mathrm{n}=1) ; \mathrm{RT}(\mathrm{n}=4) ; \mathrm{IM}(\mathrm{n}=1)$.

Observou-se que para níveis de hemoglobina mais baixos houve maior prevalência de fadiga $(p=0,030)$. Enquanto apenas $28,4 \%$ das mulheres com niveis de hemoglobina adequados estavam fatigadas, o mesmo ocorreu em 47,2\% daquelas com anemia (tabela 3 ). 
TABela 3. Razão de prevalência de fadiga segundo variáveis clínicas (São Paulo, 2007- 2008)

\begin{tabular}{|c|c|c|c|c|}
\hline Variáveis & Total & $\begin{array}{l}\text { Fadiga } \\
\text { n. }{ }^{\text {(prevalên- }} \\
\text { cia) }\end{array}$ & RP (IC 95\%) & Valor de $p$ \\
\hline $\begin{array}{c}\text { Prejuizo de sono } \\
(0-10)\end{array}$ & & & & 0,028 \\
\hline 0 & 84 & $18(21,4)$ & 1,00 & \\
\hline $1-3$ & 12 & $4(33,3)$ & $1,56(0,63-3,83)$ & \\
\hline $4-6$ & 38 & $18(47,4)$ & $2,21(1,30-3,76)$ & \\
\hline $7-10$ & 29 & $12(41,4)$ & $1,93(1,06-3,51)$ & \\
\hline $\operatorname{Dor}(0-10 ; n=162)$ & & & & 0,001 \\
\hline 0 & 86 & $14(16,3)$ & 1,00 & \\
\hline $1-3$ & 22 & $11(50,0)$ & $3,07(1,62-5,81)$ & \\
\hline $4-6$ & 40 & $19(47,5)$ & $2,92(1,63-5,22)$ & \\
\hline $7-10$ & 14 & $7(50,0)$ & $3,07(1,51-6,26)$ & \\
\hline Depressão (0 - 63) & & & & $<0,001$ \\
\hline Sem & 134 & $30(22,4)$ & 1,00 & \\
\hline Disforia & 13 & $12(92,3)$ & $4,12(2,90-5,87)$ & \\
\hline Depressão & 16 & $10(62,5)$ & $2,79(1,70-4,58)$ & \\
\hline $\begin{array}{l}\text { Capacidade funcional } \\
(10-100)\end{array}$ & & & & $<0,001$ \\
\hline $90-100$ & 120 & $28(23,3)$ & 1,00 & \\
\hline$<90$ & 43 & $24(55,8)$ & $2,39(1,57-3,64)$ & \\
\hline
\end{tabular}

RP: razão de prevalência.

Todas as variáveis clinicas analisadas apresentaram associação com significância estatística à fadiga. As pacientes com prejuízo do sono, dor, sintomas depressivos e capacidade funcional reduzida mostraram maior ocorrência de fadiga (tabela 4).

TABela 4. Estimativas da razão de prevalência de fadiga em mulheres com câncer de mama pelo modelo de regressão múltipla de Cox (São Paulo, 2007-2008)

\begin{tabular}{|l|l|l|l|}
\hline \multicolumn{1}{|c|}{ Variáveis } & \multicolumn{1}{|c|}{$\mathbf{R P}_{\mathbf{B R}}$} & \multicolumn{1}{c|}{$\mathbf{R P}_{\text {aj }}$ (IC 95\%) } & \multicolumn{1}{c|}{ Valor de $\mathbf{p}$} \\
\hline Depressão (IDB) & 1,06 & $1,06(1,03-1,09)$ & $<0,001$ \\
\hline Dor & 1,18 & $1,12(1,04-1,21)$ & 0,002 \\
\hline
\end{tabular}

$\mathrm{RP}_{\mathrm{BR}}$ : razão de prevalência bruta; $\mathrm{RP}_{\mathrm{aj}}$ : razão de prevalência ajustada; IDB: Inventário de Depressão de Beck. 
Observou-se que para um aumento de uma unidade no escore de depressão, houve aumento de $6 \%$ na chance de fadiga e, além disso, para um aumento de uma unidade na escala de intensidade de dor, o aumento na chance de fadiga foi de $12 \%$. As variáveis: nível de hemoglobina, estadiamento clínico, prejuízo do sono e capacidade funcional não apresentaram significância estatística no modelo final.

\section{Discussão}

Para identificar a prevalência de fadiga, no presente estudo, adotou-se o ponto de corte $\geq 4(0-10)$, visto que tal ponto de corte permite análise do sintoma clinicamente relevante. Outros estudos sobre o tema têm adotado o mesmo ponto de corte, o que facilita comparações (4,15-18).

Das 163 pacientes com câncer de mama que compuseram a amostra, 49,7\% apresentaram algum grau de fadiga $(\geq 1)$. Escores $\geq 4$ ocorreram em $31,9 \%$ da amostra, evidenciando a prevalência de mulheres com fadiga clinicamente relevante no presente estudo.

No presente estudo, dentre as pacientes que apresentaram algum grau de fadiga, o sintoma teve intensidade média de 6,0, o que indica fadiga moderada. Outros estudos, que avaliaram a fadiga numa escala de 0 a 10, encontraram resultado semelhante na avaliação de mulheres em tratamento adjuvante (19) e em pacientes com câncer colo-retal (20).

No entanto, notou-se grande variação da presença de fadiga, em investigações que utilizaram o mesmo ponto de corte na avaliação do sintoma $(\geq 4)$. Durante a radioterapia, apenas $13 \%$ das pacientes referiram fadiga (15) e, em pacientes que realizaram terapia hormonal (4) o resultado foi semelhante $(15,2 \%)$. Em pacientes antes do início de quimioterapia adjuvante, a prevalência de fadiga foi de $28 \%$ (16), mas em pacientes livres do câncer de mama, fadiga esteve presente em 41\% (17) e 66,1\% das amostras (18).

Dentre as variáveis relacionadas ao tumor e à terapêutica, apenas nivel de hemoglobina apresentou associação estatisticamente significativa com fadiga ( $p<0,030)$, ou seja, mulheres com baixos niveis de hemoglobina apresentaram chance $66 \%$ maior de apresentar fadiga, comparadas aquelas com niveis adequados (tabela 2 ).

Anemia é freqüente em pacientes com câncer, pois o câncer pode contribuir para a queda dos níveis de hemoglobina, pela invasão de órgãos, sangramentos tumorais, distúrbios de absorção e insuficiência da medula óssea. $\mathrm{Na}$ literatura, encontrou-se apenas um estudo que avaliou a hemoglobina em mulheres com câncer de mama durante quimioterapia adjuvante (21) e o nivel de hemoglobina observado foi semelhante ao de nosso estudo, variando de $12,1(\mathrm{DP}=1,3)$ a $12,6(\mathrm{DP}=1,3)$. A associação encontrada entre nível de hemoglobina e fadiga é coerente, visto que menor número de glóbulos vermelhos resulta em menor transporte de oxigênio para a célula e maior fadiga.

As variáveis, nível de hemoglobina, capacidade funcional, depressão, dor e prejuízo do sono, foram identificadas como possíveis fatores de risco para a fadiga em mulheres com câncer de mama e, para identificar se seriam fatores independentes, foram submetidas à análise de regressão múltipla. Para essa análise, as variáveis foram inseridas uma a uma no modelo de 
regressão e observou-se a significância estatística e a razão de prevalência das mesmas. A inserção das variáveis no modelo seguiu a ordem decrescente dos valores de $p$ : iniciou-se com depressão, seguida de dor, capacidade funcional, prejuízo do sono e nivel de hemoglobina. Os fatores independentemente associados à fadiga em mulheres com câncer de mama foram dor e depressão, pois as demais variáveis perderam significância estatística.

As mulheres com dor apresentaram chance 1,12 vezes maior de ter fadiga (tabela 4), ou seja, chance $12 \%$ maior em comparação àquelas pacientes sem dor. Dor também foi preditor de fadiga em outros estudos de pacientes com câncer de mama que utilizaram análise de regressão múltipla $(17,18)$.

As mulheres com depressão apresentaram chance 1,06 vezes maior de ter fadiga (tabela 4), ou seja, chance $6 \%$ maior em comparação às pacientes sem essa morbidade. Depressão também foi fator preditor de fadiga em outros estudos em pacientes com câncer de mama $(5,22)$.

Analisando a força das variáveis fadiga e dor sobre a fadiga, no presente estudo, é possivel identificar que a Escala Numérica de Avaliação de Dor possui 11 itens (variando de 0 a 10), ou seja, a mudança de um ponto na escala aumenta em $12 \%$ a chance de fadiga. Já na escala de depressão, que possui 64 itens (variando de 0 a 63), a mudança de um ponto no escore, aumenta em $6 \%$ a chance de fadiga. Logo, a depressão torna-se ainda mais importante na modificação da fadiga em comparação à dor.

A análise de regressão múltipla permite identificar as variáveis independentemente importantes para a ocorrência do desfecho fadiga, excluindo-se aquelas que parecem importantes para o fenômeno, mas que de fato estão "sobrepostas" a outras.

A exclusão da variável nível de hemoglobina talvez possa ser explicada pelo fato de que apenas $1 / 4$ da amostra apresentou níveis de hemoglobina abaixo dos valores de normalidade, embora entre as pacientes com anemia 47,2\% referiram fadiga. Apesar da elevada prevalência de fadiga nas pacientes com prejuízo do sono, no presente estudo, a variável não se manteve no modelo. Contudo, sono foi preditor de fadiga em outras publicações em mulheres com câncer de mama $(18,22)$.

Capacidade funcional reduzida associou-se a fadiga mais intensa em estudo de pacientes com câncer em diferentes sítios (23). No entanto, no presente estudo, o mesmo não ocorreu, o que pode ser atribuído ao fato de que apenas $1 / 4$ da amostra apresentou capacidade funcional reduzida, apesar de que, nessas pacientes, fadiga foi prevalente (tabela 3).

\section{Conclusão}

A prevalência de fadiga clinicamente significativa em mulheres com câncer de mama foi elevada e, dentre as fatigadas, a maioria apresentou fadiga de moderada a intensa. Diversos fatores clínicos mostraram associação com fadiga, confirmando a complexidade do sintoma e a existência de cluster de sintomas em oncologia. No entanto, os fatores independentemente associados à fadiga foram dor e depressão. A chance de apresentar fadiga foi $6 \%$ maior nas pacientes com depressão e $12 \%$ maior naquelas com dor. 
Dor e depressão são passíveis de tratamento na prática clínica, o que talvez possa aliviar a fadiga, visto que há poucas intervenções bem estabelecidas para o controle desse sintoma em pacientes com câncer.

O presente estudo, pioneiro em nosso meio, caracterizou a prevalência e identificou os fatores independentemente associados à fadiga em mulheres com câncer de mama e os resultados encontrados podem contribuir para o aperfeiçoamento da assistência a essa clientela.

\section{Conflito de interesse}

Este manuscrito representa um trabalho original cujo conteúdo integral ou parcial ou substancialmente semelhante não foi publicado ou submetido à publicação em outro periódico, seja no formato impresso ou eletrônico. Não há qualquer conflito de interesse dos autores em relação a este manuscrito.

\section{Apoio financeiro}

O estudo recebeu apoio financeiro da Coordenação de Aperfeiçoamento de Nivel Superior (CAPES).

\section{Referēncias}

1. Bower JE. Behavioral symptoms in patients with breast cancer and survivors. J Clin Oncol. 2008;26(5):768-77.

2. Lamino DA, Mota DDCF, Pimenta CAM. Prevalence and comorbidity of pain and fatigue in women with breast cancer. Rev Esc Enferm USP. 2011;45(2):508-14.

3. De M Alcântara-Silva TR, Freitas-Junior R, Freitas NMA, Machado GDP. Fatigue related to radiotherapy for breast and/or gynaecological cancer: a systematic review. J Clin Nurs. 2013:8.

4. Huang X, Zhang Q, Kang X, Song Y, Zhao W. Factors associated with cancer-related fatigue in breast cancer patients undergoing endocrine therapy in an urban setting: a cross-sectional study. BMC Cancer. 2010;10:453.

5. Minton O, Alexander S, Stone PC. Identification of factors associated with cancer related fatigue syndrome in disease-free breast cancer patients after completing primary treatment. Breast Cancer Res Treat. 2012;136(2):513-20.

6. Rotonda C, Guillemin F, Bonnetain F, Velten M, Conroy T. Factors associated with fatigue after surgery in women with early-stage invasive breast cancer. Oncologist. 2013;18(4):467-75.

7. Mota DDCF, Pimenta CAM, Piper, BF. Fatigue in Brazilian cancer patients, caregivers, and nursing students: a psychometric validation study of the Piper Fatigue Scale-Revised. Support Care Cancer. 2009;17(6):645-52.

8. Karnofsky DA, Burchenal JH. The clinical evaluation of chemotherapeutic agents. In: MacLeod CM, editor. Evaluation of chemotherapeutic agents. New York: Columbia University Press; 1949. 
9. Gorenstein C, Andrade L. Validation of a Portuguese version of the beck depression inventory and the state-trait anxiety inventory in Brazilian subjects. Braz J Med Biol Res. 1996;29:453-7.

10. National Comprehensive Cancer Network (NCCN). Cancer-related fatigue: NCCN Clinical Practice Guidelines in Oncology [internet]. [Citado 2010 nov 17]. Disponivel em: http://www.nccn.org.

11. Davies HT, Crombie IK, Tavakoli M. When can odds ratios mislead? BMJ. 1998;316(7136):989-91.

12. Barros AJ, Hirakata VN. Alternatives for logistic regression in crosssectional studies: in empirical comparison of models that directly estimate the prevalence ratio. BMC Med Res Methodol. 2003;3:21.

13. Lin DY, Wei LJ. The robust inference for the Cox Proportional Hazards Model. J Am Stat Assoc. 1989;84(408):1074-8.

14. Coutinho LMS, Scazufca M, Menezes PR. Métodos para estimar razão de prevalência em estudos de corte transversal. Rev Saúde Pública. 2008;42(6):992-8.

15. Lavdaniti M, Patiraki E, Dafni U, Katapodi M, Papathanasoglou E, Sotiropoulou A. Prospective assessment of fatigue and health status in Greek patients with breast cancer undergoing adjuvant radiotherapy. Oncol Nurs Forum. 2006;33(3):603-10.

16. Berger AM, Farr LA, Kuhn BR, Fischer P, Agrawal S. Values of sleep/wake, activity/rest, circadian rhythms, and fatigue prior to adjuvant breast cancer chemotherapy. J Pain Symptom Manage. 2007;33(4):398-409.

17. Meeske K, Smith AW, Alfano CM, McGregor BA, McTiernan A, Baumgartner $\mathrm{KB}$, Malone $\mathrm{KE}$, et al. Fatigue in breast cancer survivors two to five years post diagnosis: a HEAL study report. Qual Life Res. 2007;16:947-60.

18. Kim SH, Son BH, Hwang SY, Han W, Yang JH, Lee S, Yun YH. Fatigue and depression in disease-free breast cancer survivors: prevalence, correlates and association with quality of life. J Pain Symptom Manage. 2008;35(6):644-55.

19. Andrykowski MA, Donovan KA, Laronga C, Jacobsen PB. Prevalence, predictors, and characteristics of off-treatment fatigue in breast cancer survivors. Cancer. 2010;116(24):5740-8.

20. Mota DDCF, Pimenta CAM, Caponero R. Fadiga em pacientes com câncer colorretal: prevalência e fatores associados. Rev Lat-Am Enferm. 2012;20(3):[9 telas].

21. Blair S, Bardwell WA, Podbelewicz-Schuller Y, Mortimer JE. Correlation between hemoglobin and fatigue in women undergoing adjuvant chemotherapy without erythropoietin-stimulating-agent support. Clin Breast Cancer. 2008;8(6):522-6.

22. Dhruva A, Dodd M, Paul SM, Cooper BA, Lee K, West C, et al. Trajectories of fatigue in patients with breast cancer before, during, and after radiation therapy. Cancer Nurs. 2010;33(3):201-12.

23. Cheng KK, Lee DT. Effects of pain, fatigue, insomnia, and mood disturbance on functional status and quality of life of elderly patients with cancer. Crit Rev Oncol Hematol. 2011;78(2):127-37. 